\title{
THE OS ELIXIR: ON THE IMPACT OF OPERATING SYSTEM UPGRADES ON MOBILE DEVICE LIFESPAN
}

\author{
A Thesis by
}

Vinod Ramavadh Yadav

Bachelor of Electronics and Telecommunication, University of Mumbai, India, 2012

Submitted to the Department of Electrical Engineering and Computer Science and the faculty of the Graduate School of

Wichita State University

in partial fulfillment of

the requirements for the degree of

Master of Science

May 2015 
(C) Copyright 2015 by Vinod Ramavadh Yadav

All Rights Reserved 


\section{THE OS ELIXIR: ON THE IMPACT OF OPERATING SYSTEM UPGRADES ON MOBILE DEVICE LIFESPAN}

The following faculty members have examined the final copy of this thesis for form and content, and recommend that it be accepted in partial fulfillment of the requirement for the degree of Master of Science with a major in Computer Networking.

Vinod Namboodiri, Committee Chair

Abu Assaduzaman, Committee Member

Atri Dutta, Committee Member 


\section{DEDICATION}

To my parents, my brothers and my dear friends 
You will never know until you TRY. 


\section{ACKNOWLEDGEMENTS}

I would like to express my special appreciation and thanks to my advisor, Dr. Vinod Namboodiri, for his excellent guidance, caring, patience, and providing me with an excellent atmosphere for doing research. His advice on both research as well as on my career has been priceless. His guidance helped me throughout the research.

I would also like to thank my parents for their encouragement, support and having faith in me. I am also grateful to all my friends who supported me and cheered me up during all the times. 


\begin{abstract}
With the rapid proliferation of mobile devices, the issues of energy consumed to manufacture these devices and the environmental impact of electronic waste comes to the fore. One avenue to improve upon these issues is to increase the lifespan of these devices. Increase in mobile device lifespan can be achieved only if consumers retain their devices longer which could be accomplished by a combination of changes in hardware manufacturing processes, software upgrades, economic incentives, education, to name a few. The goal of this work is to specifically study the impact of operating system (OS) software upgrades on increase in mobile device lifespan. This work attempts to quantify how OS upgrades can bridge the performance gap between older devices and newer devices and motivate users to retain their devices longer. This work takes the approach of employing benchmarking tools to compare the performance of an older device to a newer device before and after an OS upgrade. Multiple devices and user applications are empirically evaluated to arrive at generalizable conclusions beginning with a conceptual framework to quantify the impact of OS upgrades.
\end{abstract}




\section{TABLE OF CONTENTS}

Chapter

Page

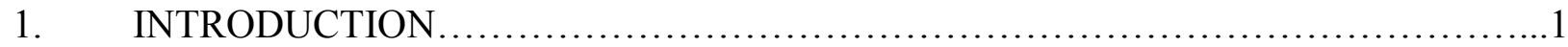

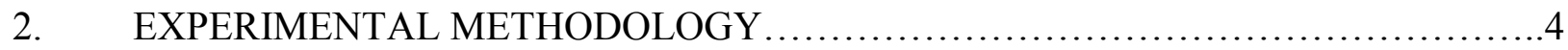

$2.1 \quad$ Overview.............................................................

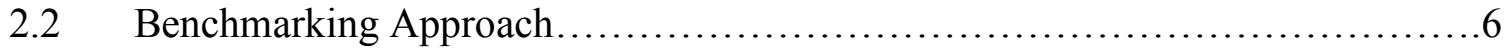

2.3 Application Profiles and Component Weights............................... 7

$2.4 \quad$ Devices and Oss................................................................

3. EVALUATION RESULTS ................................................. 11

3.1 Raw Benchmark Scores..............................................11

3.1.1 Results from primary pair...................................... 12

3.1.2 Results from other devices........................................... 14

3.2 Application Performance Scores....................................... 19

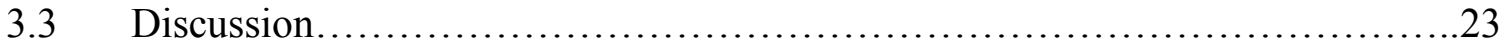

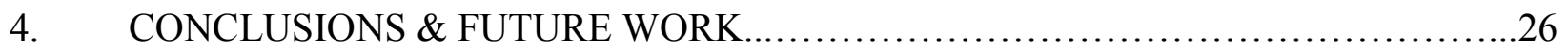

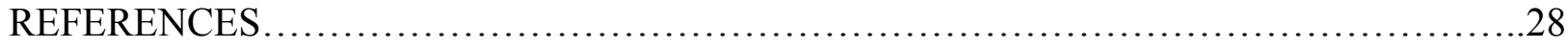




\section{LIST OF TABLES}

Table

Page

1. Application Profiles and Test Methodology......................................

2. Hardware Component Weights for Various Application Profiles.......................9

3. Devices studied for impact of OS upgrades...................................... 11 


\section{LIST OF FIGURES}

Figure

Page

1. Concept of performance gaps when evaluating benefit of OS upgrades on

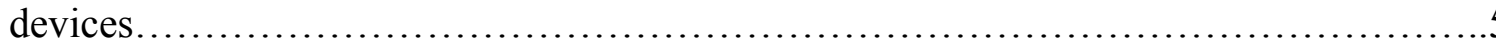

2. Impact of OS upgrade on overall performance scores for HTC Desire compared to Samsung S7562 ................................................ 12

3. Impact of OS upgrade on individual component performance scores for HTC Desire compared to Samsung S7562 .................................... 13

4. Impact on OS upgrades on overall performance scores for HTC Desire and

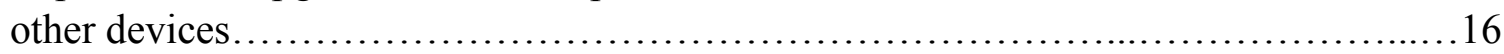

a. HTC Desire and Sony Xperia............................................ 15

b. HTC Desire and HTC SV ............................................. 15

c. HTC Desire and Intel Xolo............................................ 16

5. Impact on OS upgrades on individual component performance scores for HTC Desire and other devices................................................ 18

a. HTC Desire and Sony Xperia........................................ 17

b. HTC Desire and HTC SV ............................................. 17

c. HTC Desire and Intel Xolo........................................... 18

6. Impact on OS upgrades on various applications................................22

a. Audio application..................................................

b. Streating Audio application........................................... 20

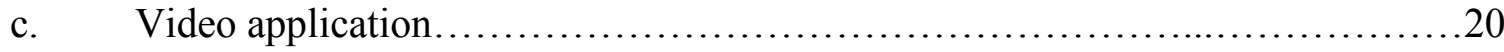

d. Streaming Video application..........................................21

e. Email application.....................................................21

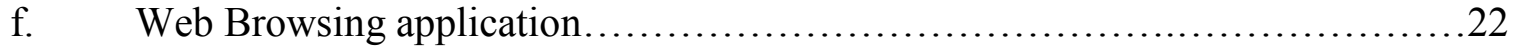

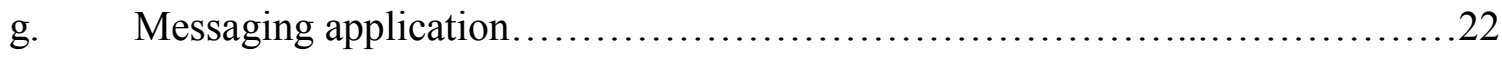

7. Illustration that OS upgrade benefits are limited to only a few versions before hardware requirements are not met. ... 


\section{LIST OF ABBREVIATIONS / NOMENCLATURE}

NN

$\mathrm{ON}$

$\mathrm{OO}$

OS
New device New operating system

Old device New operating system

Old device Old operating system

Operating System 


\section{CHAPTER 1}

\section{INTRODUCTION}

When it comes to examining energy efficiency and the concept of environmental sustainability in computing, the focus has invariably been on data centers and mobile infrastructures like cell towers, because they have been considered the power hogs within the sector. There has, however, been a big shift in how the general population performs computing and communication tasks these days with a much greater reliance on mobile, battery-operated devices. The increased role of mobile devices has resulted in recent work advocating environmental sustainability in mobile computing (e.g. [1]). The work in [2, 3] found that computing devices, including data centers, server farms, desktops, and mobile devices (laptops and mobile phones), accounted for about 3-7\% of the global electricity usage. Surprisingly, mobile devices were responsible for at least $5-10 \%$ of this share due to their large scale use, and this share is expected to grow as power-hungry smart phones proliferate the market [4]. Energyrelated research in the fast growing mobile computing area, unfortunately from an environmental perspective, has been focused primarily in addressing only the battery-lifetime issue. While optimizing battery lifetime can be useful in reducing the overall energy costs of mobile devices, it only considers energy consumed to operate these devices (i.e, use phase energy), neglecting the energy consumed to manufacture and recycle them. Mobile devices have very short lifespans compared to other computing devices (e.g. desktops), and thus, the overall energy spent (and emissions produced) in manufacturing these devices is a very significant share (upwards of 50\% [5]) of the overall life cycle. The problem of electronic waste is also an important one, with less than $10 \%$ of mobile handsets globally being recycled [5]. 
One avenue of cutting down energy costs for the non-use phase is greater efficiency in the processes (e.g. manufacturing, transportation, recycling) employed. This avenue is an ongoing approach employed by firms currently to not only cut energy costs, but also overall monetary costs. Another avenue would be increasing the lifespan of mobile devices which would cut down energy consumed for tasks like manufacturing, transportation, and recycling across the entire spectrum of mobile devices. A longer lifespan would mean that the energy consumed by a device during the non-use phases would be amortized over a longer use-phase, in turn reducing the overall energy consumed by the mobile device segment. Furthermore, the increase in lifespan option is expected to have an immediate impact and will be complementary to the ongoing search for efficiencies in processes. It has the added benefit of cutting down the rate of generation of electronic waste through a reduced rate of device replacements.

Increase in mobile device lifespan can be achieved only if consumers retain their devices longer. From a technical perspective, this would require greater emphasis on software features that can be updated, and hardware components that can be replaced to keep up (or stay close) with improved user-experience offered by newer devices. Some recent efforts that have sprung up in this direction include the yet to be released ZTE Eco-Mobius phone, and Project Ara [6], an effort from Google that includes a collaboration with Phonebloks [7]. These efforts are building modular smartphones as "blocks" which can be swapped to reduce the need for replacing the entire device. Additional approaches to consider include providing economic incentives to consumers to retain their devices longer and educating consumers on the environmental impact of frequent device replacements. In prior work in [8], the authors have surveyed a large user pool on what factors motivate the replacement of mobile phones in 1-2 years, and how economic incentives can be used to increase mobile device lifespan $[9,10]$. 
Among the possible approaches to increase mobile device lifespan, this work specifically looks at the impact of operating system (OS) software upgrades. The broad question investigated is "Can OS upgrades help improve mobile device lifespan?". Towards this broad goal, this work quantifies how OS upgrades can bridge the performance gap between older devices and newer devices and motivate users to retain their devices longer and thus increase device lifespan. The devices specifically under study will be mobile phone devices as they tend to have a high turnover rate, possibly induced as a result of frequently introduced software and hardware features, short wireless carrier contracts, and the customer's attraction of the latest device. For this study, five different phones (over a wide range of release dates) were compared in terms of performance along with seven common user application scenarios to make generalizable conclusions. The results from the extensive empirical evaluations indicate that OS upgrades can help an older device achieve comparable performance to a newer device if the latter does not benefit from a major technological leap that aids performance. 


\section{CHAPTER 2}

\section{EXPERIMENTAL METHODOLOGY}

This chapter describes the methodology behind studying the impact of OS upgrades on bridging the performance gap between older devices and newer devices.

\subsection{Overview}

The objective of the experiments performed are to determine by how much an older device's performance improves by upgrading its OS and then comparing it to a newer device and studying the performance gap between the older device with a new OS and a newer device with the same OS. Consider the scenario of two devices and two OS versions. With the letter O signifying an older device or OS, and N signifying a newer device or OS, the tuple of $\{$ device, $O S\}$ would have four possible pairings $\{\{\mathrm{O}, \mathrm{O}\},\{\mathrm{O}, \mathrm{N}\},\{\mathrm{N}, \mathrm{O}\},\{\mathrm{N}, \mathrm{N}\}\}$. The scenario of an old OS on a new device is of no interest as does not promise any benefits to a new device compared to the newer OS. Thus, the three scenarios of interest, with simplified notation are the set $\{\mathrm{OO}, \mathrm{ON}, \mathrm{NN}\}$. The objective of this work is to compare these three device and OS pairings to make a determination of the benefit of OS upgrades on a mobile device.

The difference in performance between the new and old OS on the old device, i.e. OO and ON, can be termed as the "OS gap" which is the performance benefit added to the old device when the OS is upgraded from the old to the new. The difference in performance between ON

and NN can be termed as the "device gap" which is the performance benefit added when the old device (with the new OS) is replaced with the new device. Finally, the difference in performance between $\mathrm{OO}$ and $\mathrm{NN}$ can be termed as the "replacement gap" which is the performance benefit gained by moving to a new device and OS from the old device with the old OS. 
This work studies these three performance gaps for multiple device and OS pairings and uses these results to comment on under what conditions OS upgrades can improve device lifespan. OS upgrades can help increase device lifespan when the device gap is small; that is, the performance benefits of changing to a new device is small after an OS upgrade on an older device. In other words, lifespan increase can be expected when an OS upgrade bridges most of the replacement gap.

As shown in Figure 1, is the conceptual schematic of the device and OS pairings and the OS gap, the device gap, and the replacement gap.

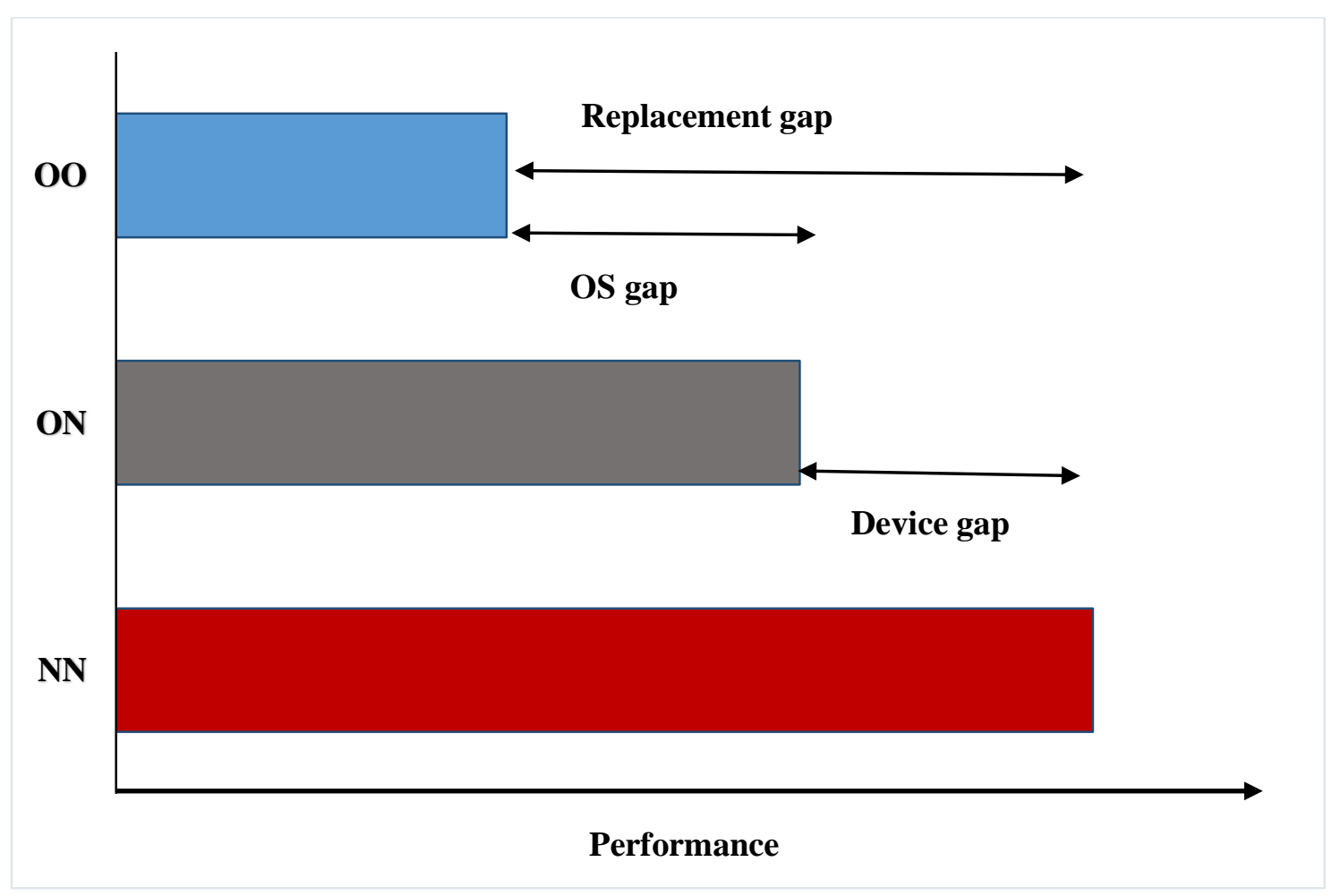

Figure 1. Concept of performance gaps when evaluating benefit of OS upgrades on devices. 


\subsection{Benchmarking Approach}

The Passmark Performance Testing tool for Android [11] was used for benchmarking performance of devices with a specific OS. This tool computes performance scores for each of the hardware components of interest and also an overall score that combines these individual scores with some weights. However, these component scores and weights used may not reflect how a user perceives performance which is dependent more on how applications perform. Thus, to better understand how the various applications would perform from a user's perspective seven different representative application profiles were picked and a weighted performance score computed based on the use of the four important hardware components: CPU, memory, disk and graphics. These components were picked because these are the ones most closely associated with system performance of a device. The CPU test of the Passmark tool involves operations with integers and floating point numbers, computation of prime numbers, string sorting, compression, and encryption tests. A higher score on this test indicates greater prowess in computational tasks and multitasking. In the disk test, memory read and write for external or internal memory is performed using the Java programming language's FileOutputStream and FileInputStream classes. In the memory test, sequential memory read and write operations are performed on RAM. Greater scores on memory and disk tests indicate lower latency in launching applications and accessing stored data. Finally, the graphics test performs both 2D and 3D tests that include drawing solid, transparent and complex vectors, and performing image rendering and filtering tasks. A higher score on this test indicates better support for graphical rendering.

Known limitations of approach other hardware components such as display, speakers, and camera are important in addition to those considered above, but cannot necessarily be measured through the benchmarking approach taken in this work. Nevertheless, the study of the 
performance of CPU, memory, disk, and graphics is still significant from the perspective of application performance which was found to be the second biggest reason (behind battery life) users replace mobile devices in a recent user survey [8]. Communications and networking components such as WiFi, Cellular, and Bluetooth are important for application performance, but were omitted as all devices considered in our study had similar capabilities for these network interfaces. An important omission in our methodology is battery performance. It is well known that battery life degrades with number of charge cycles and thus will be much poorer on an older device regardless of OS performance. It is assumed that when battery performance degrades enough on an old device to affect user satisfaction, it is replaced with a new battery. Battery replacements cost less than $10 \%$ of the original cost of the device and are increasingly an option from sites such as iCracked.com. Modular blocks such as those envisioned by Project Ara [6] will make it easy to swap batteries for any user.

\subsection{Application Profiles and Component Weights}

Seven different application profiles were created to cover a wide range of user activities on a mobile device. These application profiles are listed in Table 1 along with the specifics on how these were tested. On each device, each application profile was tested 10 times with the mean scores reported in the following section. 
As shown in Table 1, we have created seven different profiles and listed the test methodology followed to measure the utilization.

TABLE 1

APPLICATION PROFILES AND TEST METHODOLOGY

\begin{tabular}{|c|c|c|}
\hline Application Profiles & Tools & Description \\
\hline Audio from Disk & Music Player for Android & $\begin{array}{c}\text { An Audio file of size 8 MB was } \\
\text { played }\end{array}$ \\
\hline Audio Streaming Online & Slacker Radio & $\begin{array}{c}\text { The same song lasting 283 } \\
\text { seconds was played }\end{array}$ \\
\hline Video from Disk & Video Player for Android & $\begin{array}{c}\text { A Video file of size 5 MB was } \\
\text { played }\end{array}$ \\
\hline Video Streaming Online & $\begin{array}{c}\text { Default Browser at } \\
\text { www.youtube.com }\end{array}$ & $\begin{array}{c}\text { The same video lasting 273 } \\
\text { seconds was played }\end{array}$ \\
\hline Web Browsing & $\begin{array}{c}\text { Default Android Web } \\
\text { Browser }\end{array}$ & $\begin{array}{c}\text { Two Articles from www.cnn.com } \\
\text { were opened and read }\end{array}$ \\
\hline Email & Gmail & $\begin{array}{c}\text { Three emails were opened and } \\
\text { read, with two replies sent }\end{array}$ \\
\hline Messaging & Android SMS and \\
WhatsApp & $\begin{array}{c}\text { Both these application were used } \\
\text { to send 160 byte messages }\end{array}$ \\
\hline
\end{tabular}

For each application profile a weight was assigned (see Table 2) to each hardware component based on its importance to the performance of the application. The importance of a component was estimated by measuring its utilization when running the application. Utilization of components when running an application were measured through logs captured by shell scripts from the Android Monitor tool. 
As shown in Table 2, we have assigned weights to all the seven different profile we have created on basis of the utilization of CPU, DISK, MEMORY and GRAPHIC to do the task listed in test methodology table 1.

TABLE 2

HARDWARE COMPONENTS WEIGHTS FOR VARIOUS APPLICATION PROFILES

\begin{tabular}{|c|c|c|c|c|}
\hline $\begin{array}{c}\text { Application } \\
\text { Profiles }\end{array}$ & CPU & MEMORY & DISK & GRAPHICS \\
\hline Audio from Disk & 0.65 & 0.1 & 0.1 & 0.15 \\
\hline $\begin{array}{c}\text { Audio Streaming } \\
\text { Online }\end{array}$ & 0.65 & 0.08 & 0.12 & 0.15 \\
\hline Video from Disk & 0.5 & 0.1 & 0.1 & 0.3 \\
\hline $\begin{array}{c}\text { Video Streaming } \\
\text { Online }\end{array}$ & 0.55 & 0.05 & 0.15 & 0.25 \\
\hline Web Browsing & 0.45 & 0.1 & 0.1 & 0.35 \\
\hline Email & 0.58 & 0.05 & 0.12 & 0.25 \\
\hline Messaging & 0.55 & 0.08 & 0.12 & 0.25 \\
\hline
\end{tabular}

\subsection{Devices and OSs}

The devices primarily tested were the HTC Desire A8181 with Android stock OS version 2.2.2 (Froyo) and the Samsung Galaxy S Duos s7562 with stock Android OS version 4.0.4 (Ice Cream Sandwich). These devices were released in March 2010 and September 2012 respectively, with the HTC device acting as the "old" device and the Samsung device as the "new" device. These devices act as good test cases because, even though there is a gap of more than 2 years in their release dates, both have similar architectures (for example, single core CPUs).

For the older device, it was possible to test many Android OS versions such as the default 2.2.2, 2.3.3, and 4.0.4 through a vendor release of upgraded OS versions or through other 
developers releasing custom ROMs. Battery replacements cost less than $10 \%$ of the original cost of the device and are increasingly an option from sites such as iCracked.com. Modular blocks such as those envisioned by Project Ara [6] will make it easy to swap batteries for any user. 


\section{CHAPTER 3}

\section{EVALUATION RESULTS}

\subsection{Raw Benchmark Scores}

The evaluations results can be divided into two sets. The first set of results are from the two primary devices described in the previous section: HTC Desire and Samsung s7562. All the benchmark tests were performed on both devices multiple times with the mean score reported. The second set of results are based on the results of one of the devices from the first set and those reported and collected in the benchmark results repository at [13]. The use of a repository allowed comparisons too many more devices beyond the primary one used and allows making more generalized conclusions.

As shown in Table 3, we have created six different profile and listed the test methodology followed to measure the utilization.

TABLE 3

DEVICES STUDEID FOR IMPACT OF OS UPGRADES

\begin{tabular}{|c|c|c|c|c|c|}
\hline Device Model & Launch Date & CPU & Memory & Disk & GPU \\
\hline \hline HTC Desire & $\begin{array}{c}\text { February } \\
2010\end{array}$ & $\begin{array}{c}1 \mathrm{GHz} \text { Scorpion } \\
\text { ARMv7 }\end{array}$ & $576 \mathrm{MB}$ & $512 \mathrm{MB}$ & Adreno 200 \\
\hline $\begin{array}{c}\text { Sony Xperia U } \\
2012\end{array}$ & $\begin{array}{c}\text { Dual core 1 GHz } \\
\text { Cortex-A9 }\end{array}$ & $512 \mathrm{MB}$ & $8 \mathrm{~GB}$ & Mali-400 \\
\hline $\begin{array}{c}\text { Samsung GT- } \\
\text { s7562 }\end{array}$ & July 2012 & $1 \mathrm{GHz}$ Cortex-A5 & $769 \mathrm{MB}$ & $4 \mathrm{~GB}$ & Adreno 200 \\
\hline $\begin{array}{c}\text { HTC Desire } \\
\text { SV }\end{array}$ & $\begin{array}{c}\text { November } \\
2012\end{array}$ & $\begin{array}{c}\text { Dual core 1 GHz } \\
\text { Cortex-A5 }\end{array}$ & $768 \mathrm{MB}$ & $4 \mathrm{~GB}$ & Adreno 203 \\
\hline $\begin{array}{c}\text { Intel Xolo } \\
\text { X910 }\end{array}$ & May 2013 & $\begin{array}{c}1.6 \mathrm{GHz} \text { Intel Atom } \\
\text { Z2460 }\end{array}$ & $1 \mathrm{~GB}$ & $4 \mathrm{~GB}$ & $\begin{array}{c}\text { PowerVR } \\
\text { SGX540 }\end{array}$ \\
\hline
\end{tabular}




\subsubsection{Results from primary pair:}

The results presented here are in terms of an overall performance score reported by the Passmark benchmarking tool for a device and OS combination (OO, ON or NN) and also individual component (CPU, memory, disk, and graphics) scores that contribute to the overall score.

As shown in Figure 2, the overall performance of HTC Desire (old device) is compared with overall performance of Samsung s7562 (new device).

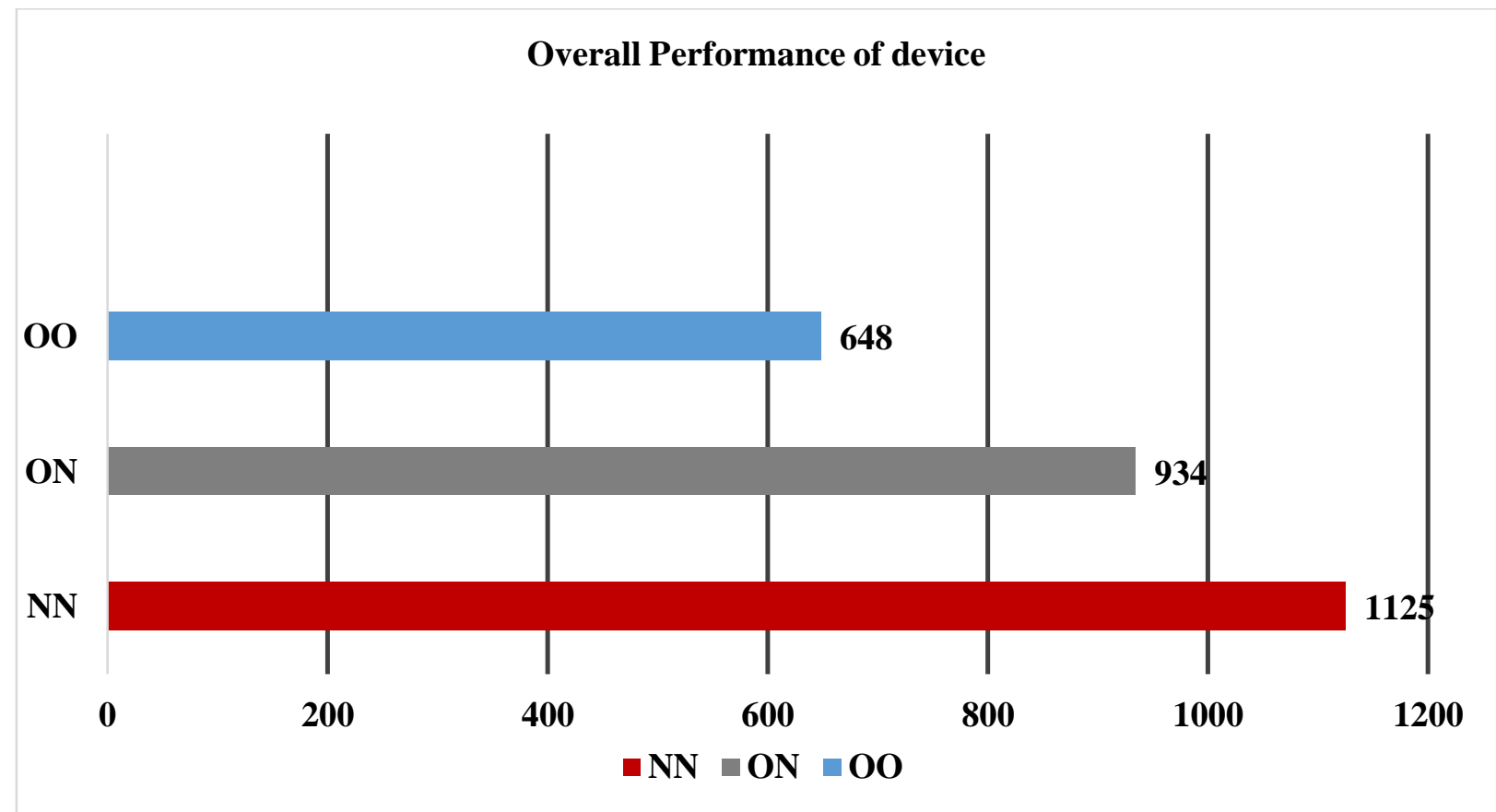

Figure 2. Impact of OS upgrade on overall performance scores for HTC Desire compared to Samsung S7562.

Figure 2 shows that with an OS upgrade the older device's performance jumps from 648 to 934, a $44.14 \%$ improvement in performance. Though the NN scenario provides an $73.6 \%$ improvement in performance score over the OO scenario (as expected), the OS upgrade scenario of $\mathrm{ON}$ reduces the replacement gap by $60 \%$. 
As shown in Figure 3, the detailed score comparison of HTC Desire and Samsung s7562 is done with respect to each hardware component.

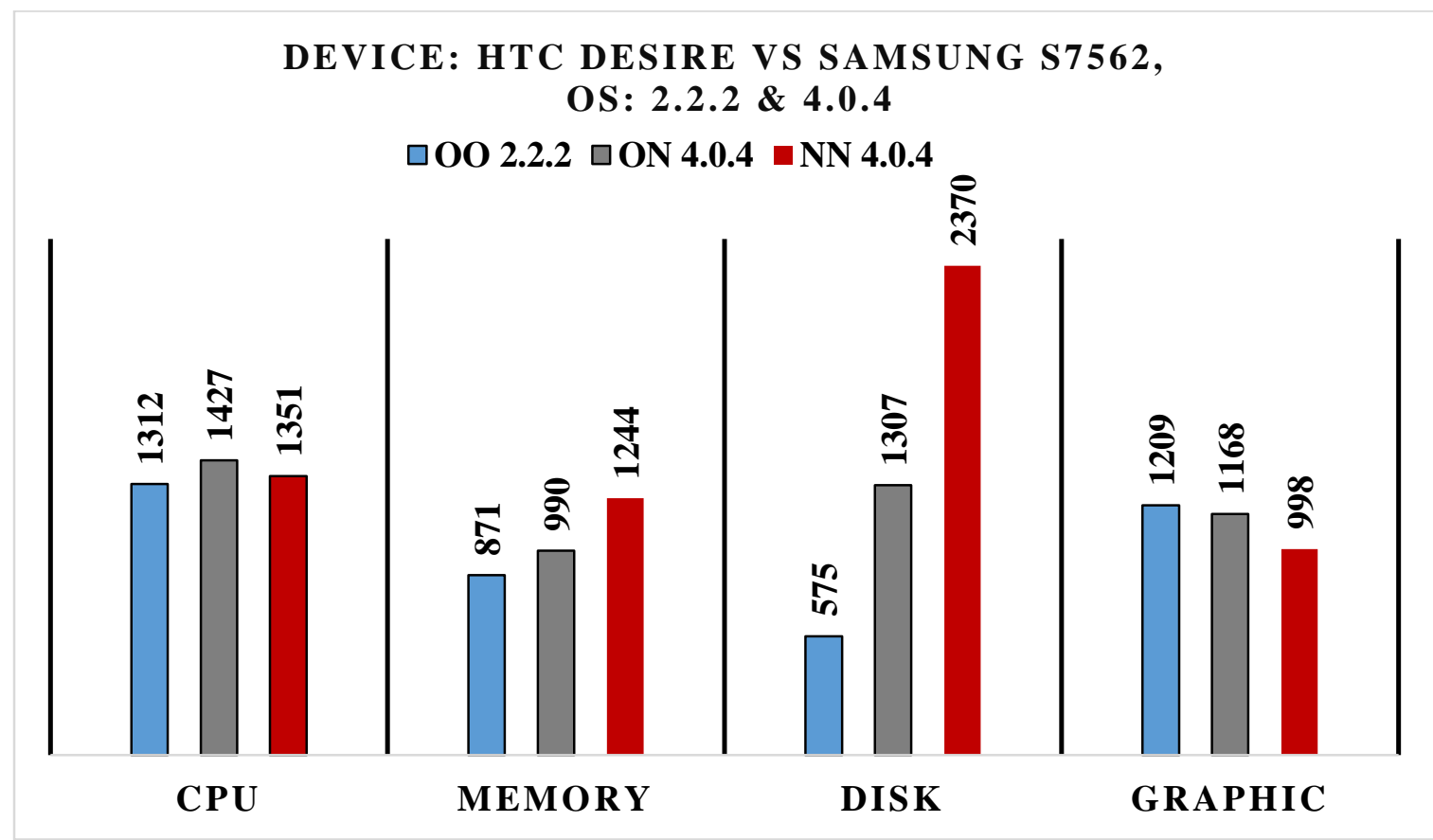

Figure 3. Impact of OS upgrade on individual component performance scores for HTC Desire compared to Samsung S7562.

Figure 3 provides a more detailed breakdown of scores across specific components of interest and gives more insight into where differences lie in terms of the overall scores seen in Figure 2. It can be seen that the performance of the disk (and to a lesser extent memory) is where the new device is much better than the old device, and that the OS upgrade seems to have significantly helped in this regard. Interestingly, the CPU scores of the $\mathrm{OO}$ and NN scenarios are very similar, and the OS upgrade on the old device helps improve the CPU score of the ON scenario to be even higher than the NN scenario. This result is seen because both the devices have very similar CPU capabilities, and the OS upgrade seems to have maximized the performance derived from the old device's CPU even more than the new device's CPU. The scores for the graphics component is also surprising where the scores decrease slightly as we go from the $\mathrm{OO}$ to the $\mathrm{ON}$ 
and finally the NN scenario. This is believed to be because the GPUs are similar for both devices and the original OS on the old devices was likely the most optimized to work with graphics.

Thus, for the device pair and OSs considered, it can be safely concluded that OS upgrades can provide a performance boost to the older device that can significantly reduce the performance edge of a newer device.

\subsubsection{Results from other devices:}

The primary pair of devices considered were released more than a year apart, but they had many components of comparable capabilities. Thus, the performance of other devices (serving as NN scenarios) were compared to that of the HTC Desire device (which we had physical access to and could get scores for the $\mathrm{OO}$ and ON scenarios). Three additional devices,

Sony Xperia, HTC SV, and the Intel Xolo were used for NN scores and compared with the HTC Desire device used for the $\mathrm{OO}$ and $\mathrm{ON}$ scenarios. These three devices were released at various points of time after the HTC Desire device at similar price points, thus making them fall on the natural mobile device technology evolution curve and making it fair to use them for comparisons. 
As shown in Figures 4a, 4b and 4c, the overall performance scores of HTC Desire is compared with three other device.

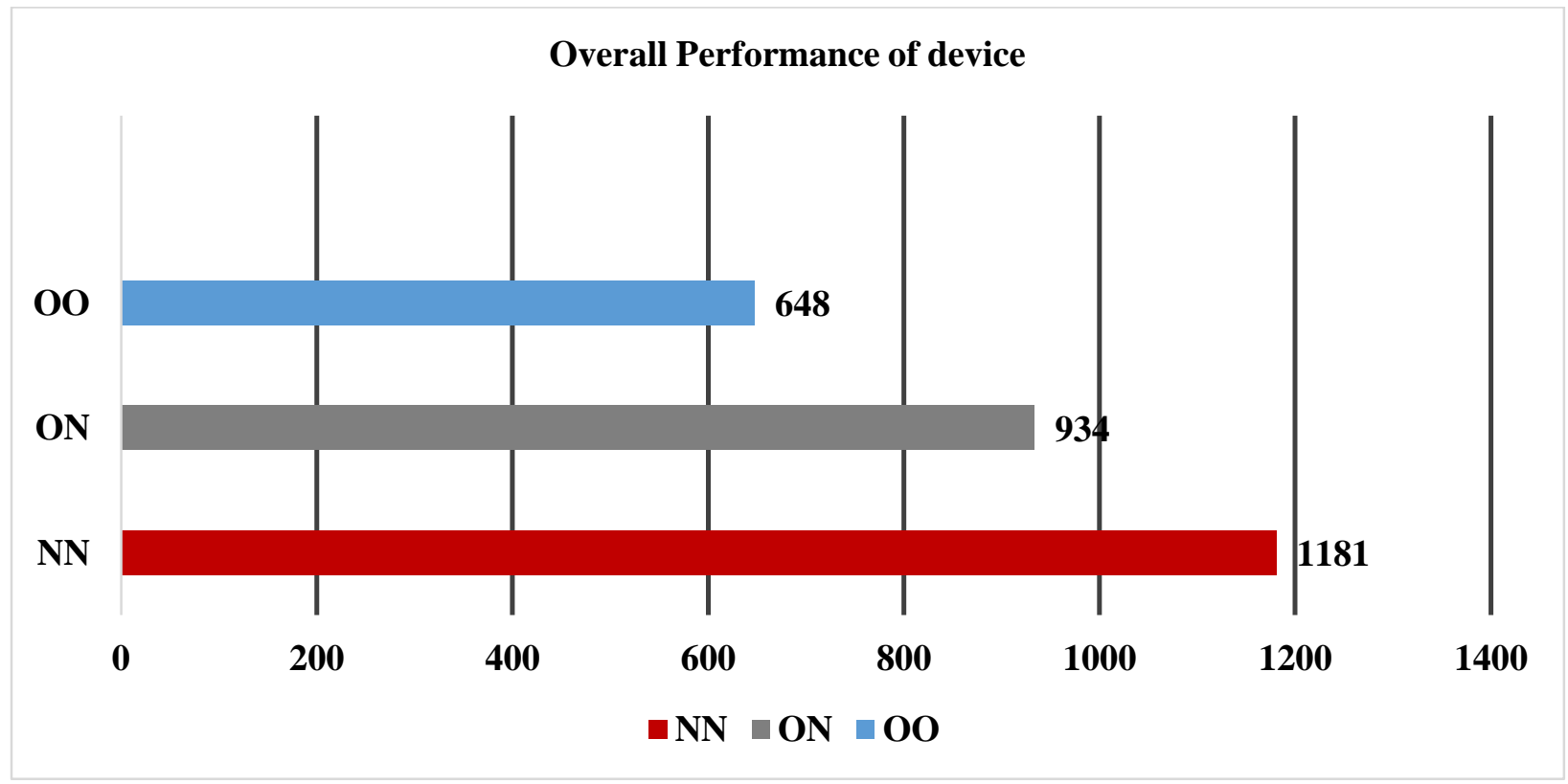

Figure 4a. HTC Desire and Sony Xperia.

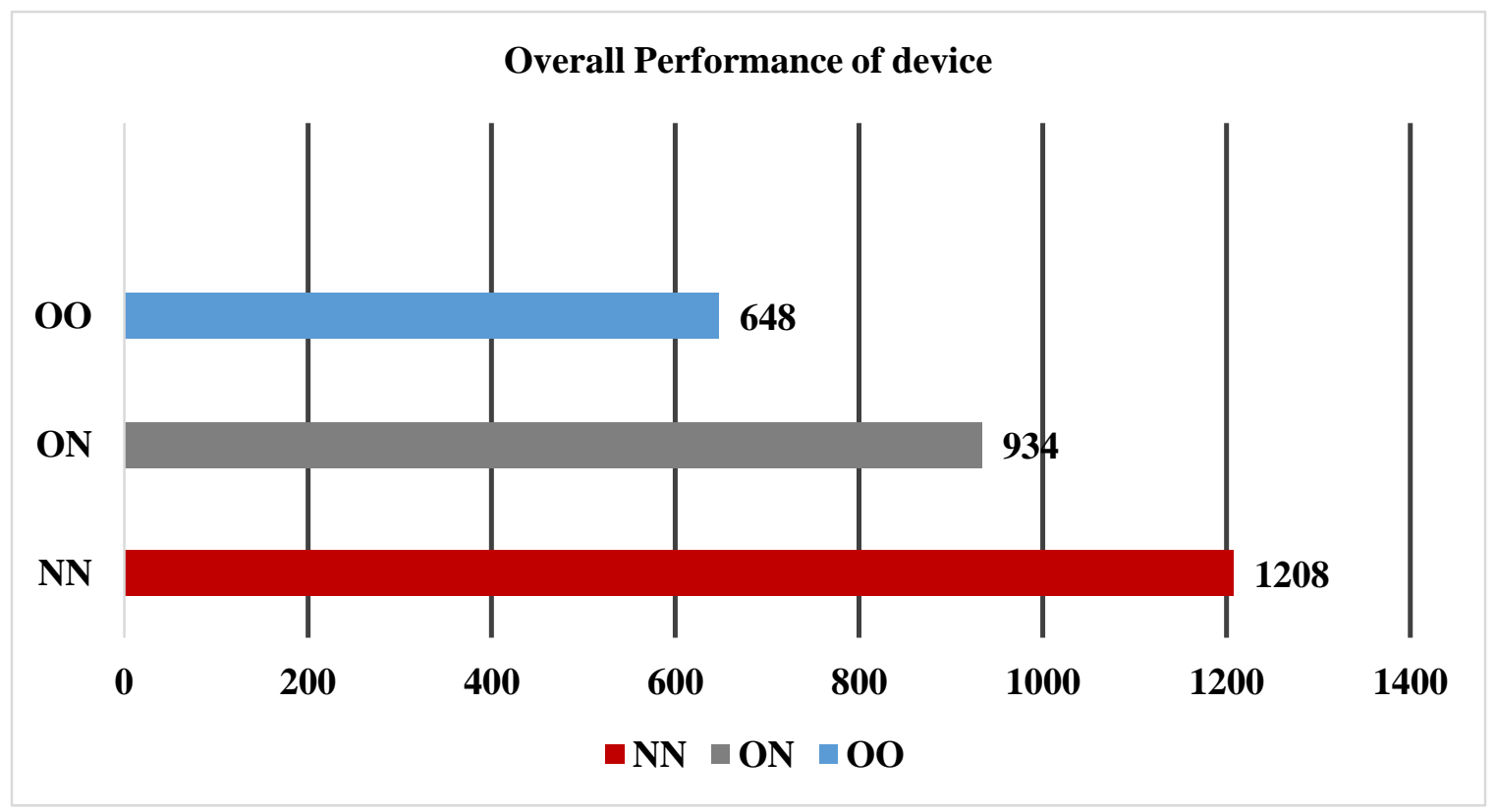

Figure 4b. HTC Desire and HTC SV. 


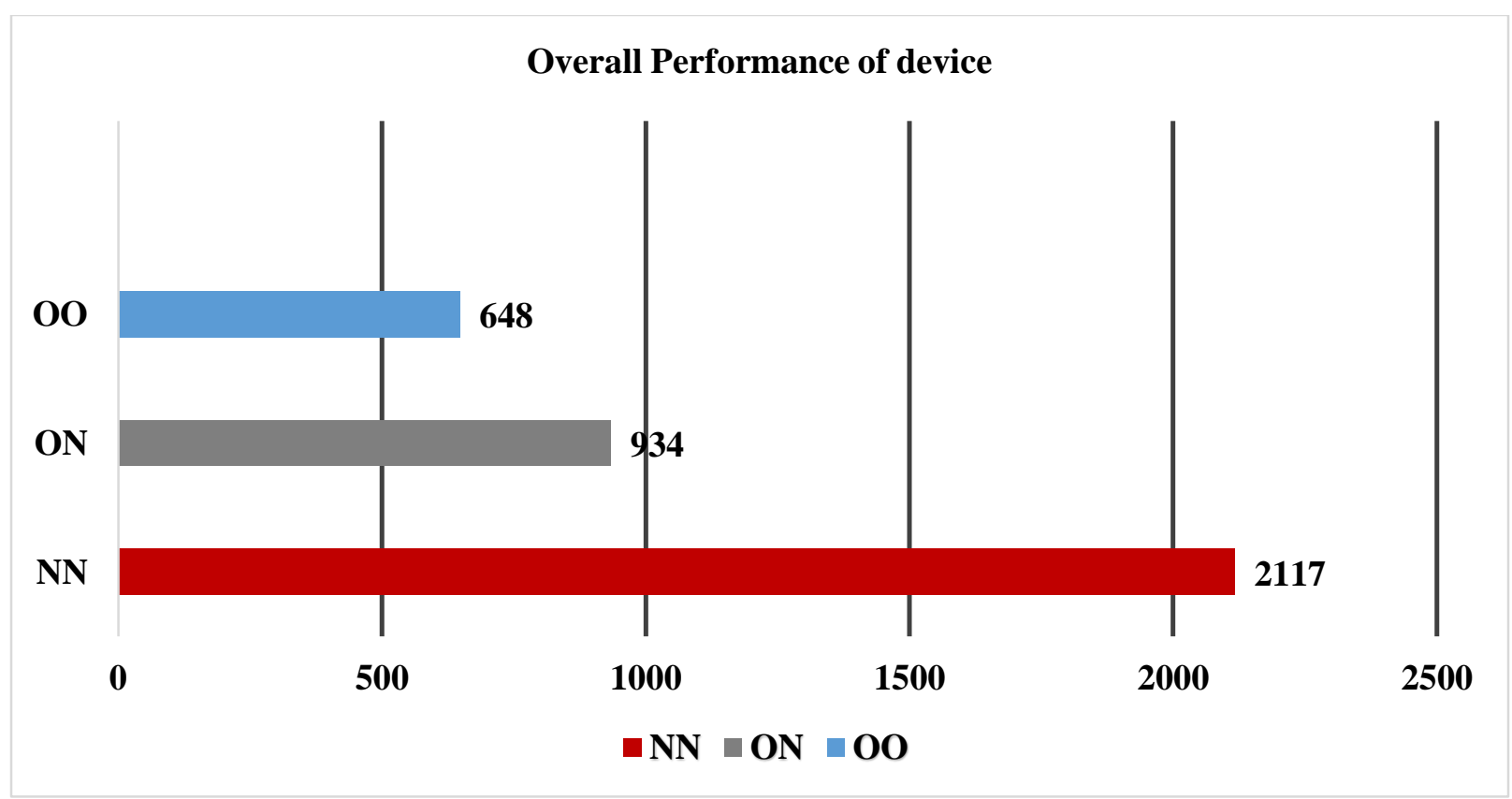

Figure 4c. HTC Desire and Intel Xolo.

Figure 4. Impact on OS upgrades on overall performance scores for HTC Desire and other devices.

It can be observed from figure 4 that the newest device Intel Xolo has the greatest replacement gap. This device used the Intel Atom CPU that goes into netbook profile computers and is much more capable than those of other devices compared. The replacement gap for the other devices released closer to that of the HTC Desire were also higher than seen in Figure 2 before, but not a big increase. 
As shown in Figure 5a, 5b and 5c, the breakdown of performance scores for each of the three devices pair are studied and compared.

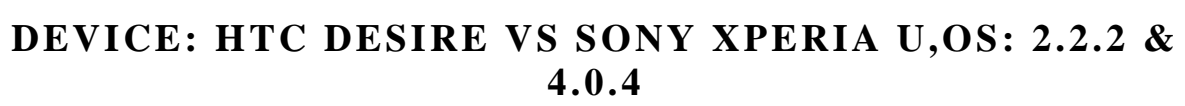

$\square O O 2.2 .2 \quad \square \mathrm{ON}$ 4.0.4 $\square \mathrm{NN}$ 4.0.4

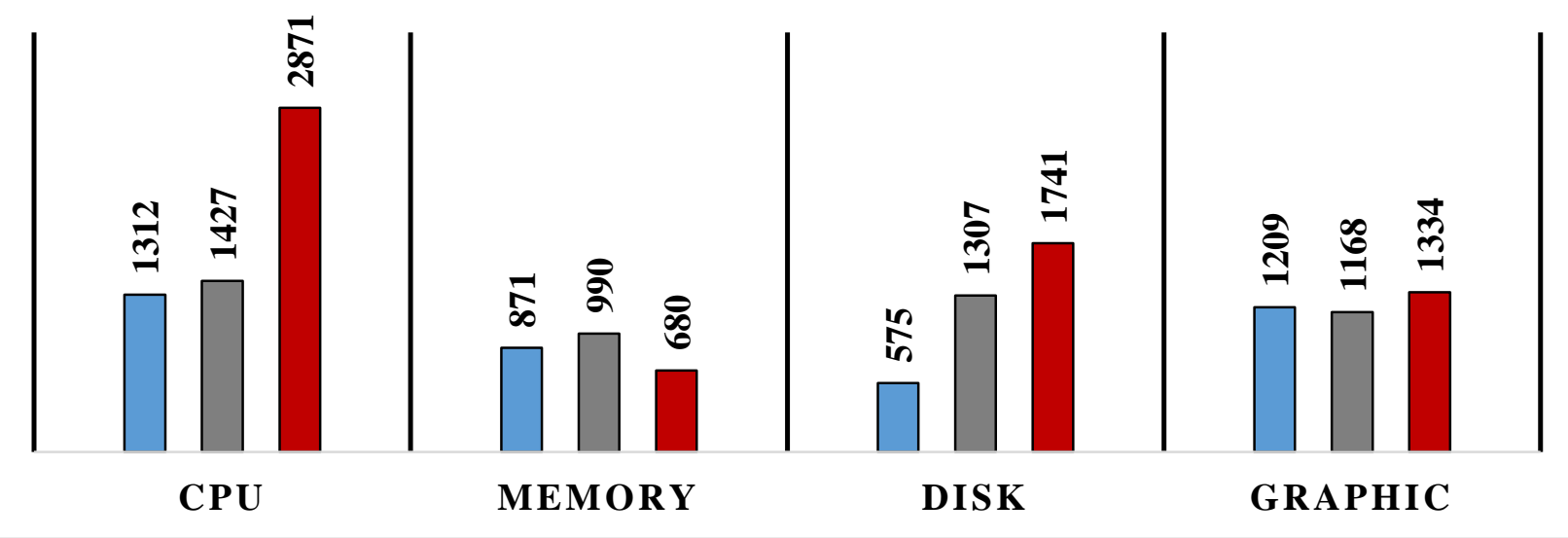

Figure 5a. HTC Desire and Sony Xperia.

DEVICE: HTC DESIRE VS HTC DESIRE SV, OS: $2.2 .2 \&$ 4.0.4

$\square O O$ 2.2.2 $\square$ ON 4.0.4 $\square$ NN 4.0.4

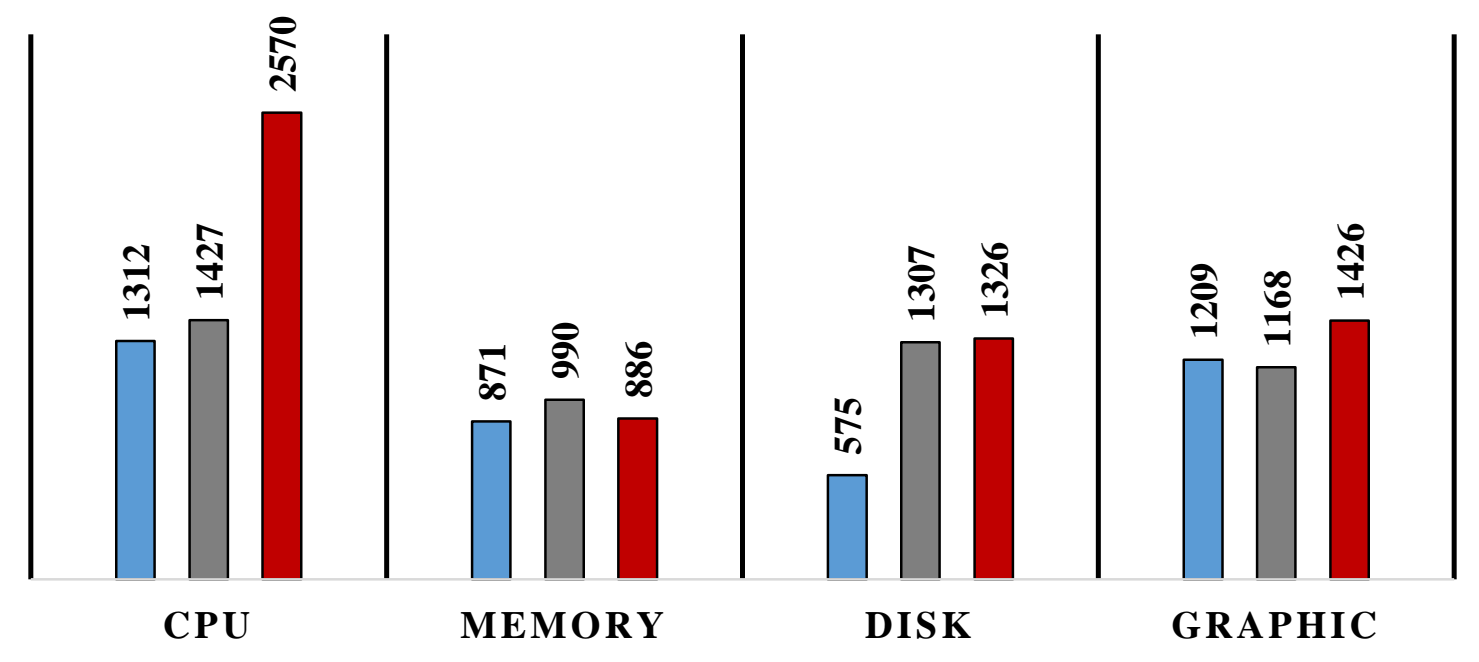

Figure 5b. HTC Desire and HTC Desire SV. 


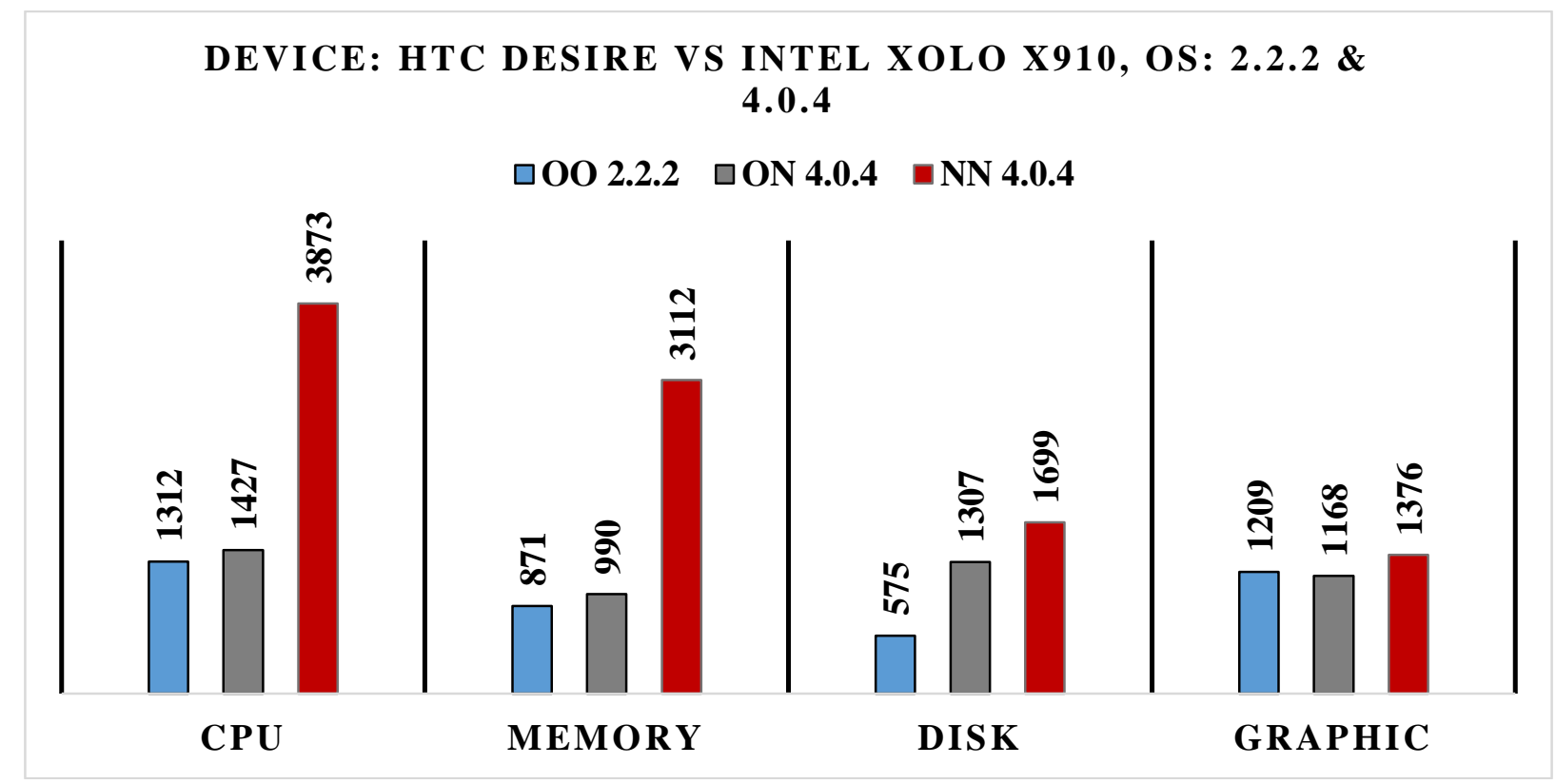

Figure 5c. HTC Desire and Intel Xolo.

Figure 5. Impact on OS upgrades on individual component performance scores for HTC Desire and other devices.

Figures 5 shows the breakdown of performance scores for each of the three device pairs. The CPU performance can be seen to be the biggest reason for the larger replacement gap. The Sony Xperia and HTC SV devices improve upon the HTC Desire by using a dual-core processor. However, the Atom processor used in Intel Xolo can truly be considered a cut above the others and represents a technological leap in smartphone CPUs compared to the prior devices.

Thus, it can be concluded from these results that OS upgrades do not meaningfully reduce the device replacement gaps when newer devices have components with significant technology leaps. However, when newer devices do not have components that are a significant leap, and OS upgrade can be very effective in minimizing the device replacement gap. 


\subsection{Application Performance Scores}

This set of experiments looked at how OS upgrades impact individual application performance (to consider a user perspective) as opposed to looking at overall scores for a device based on unknown weights assigned by the Passmark application to each hardware component. The results computed here were based on the weights presented in Table 2 .

As shown in Figure 6a, 6b, 6c, 6d, 6e, 6f and 6g, the Impact of OS Upgrades on application profiles which we have created is evaluated.

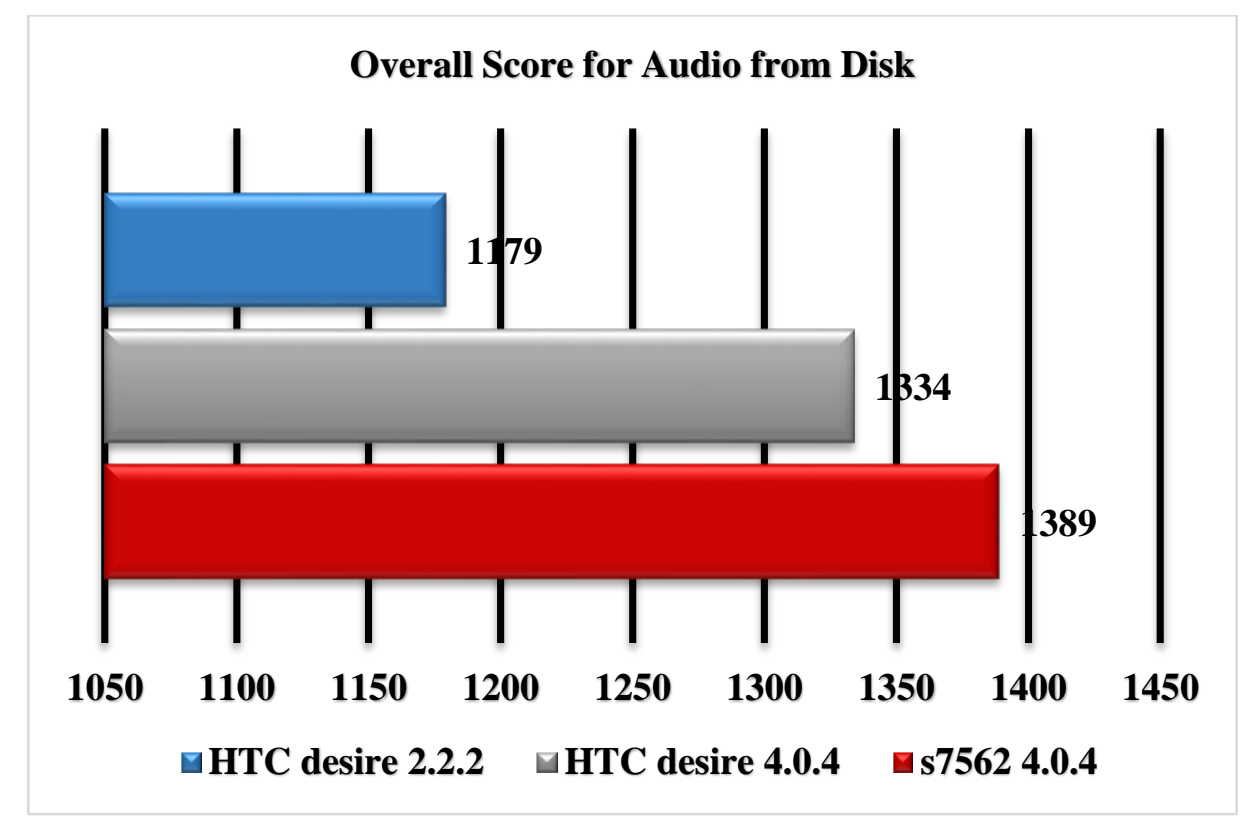

Figure 6a. Playing Audio from Disk application. 


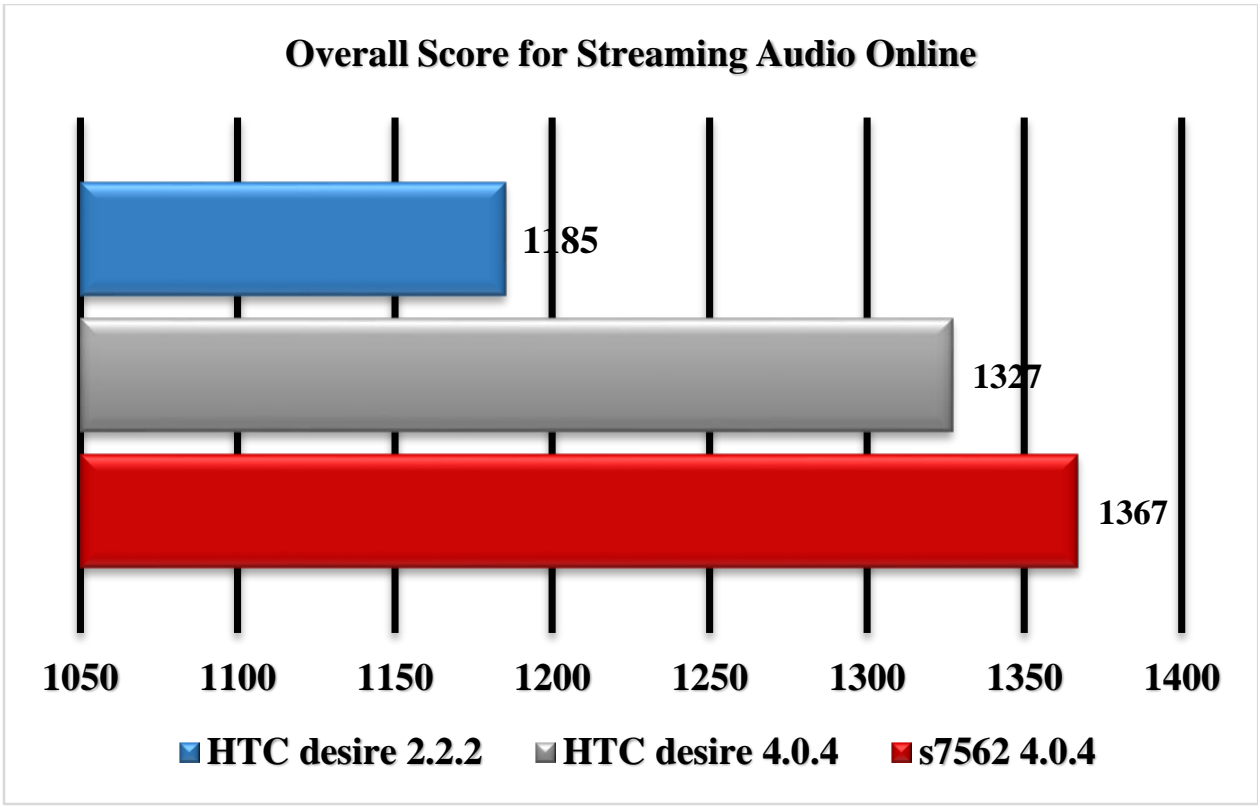

Figure 6b. Streaming Audio application.

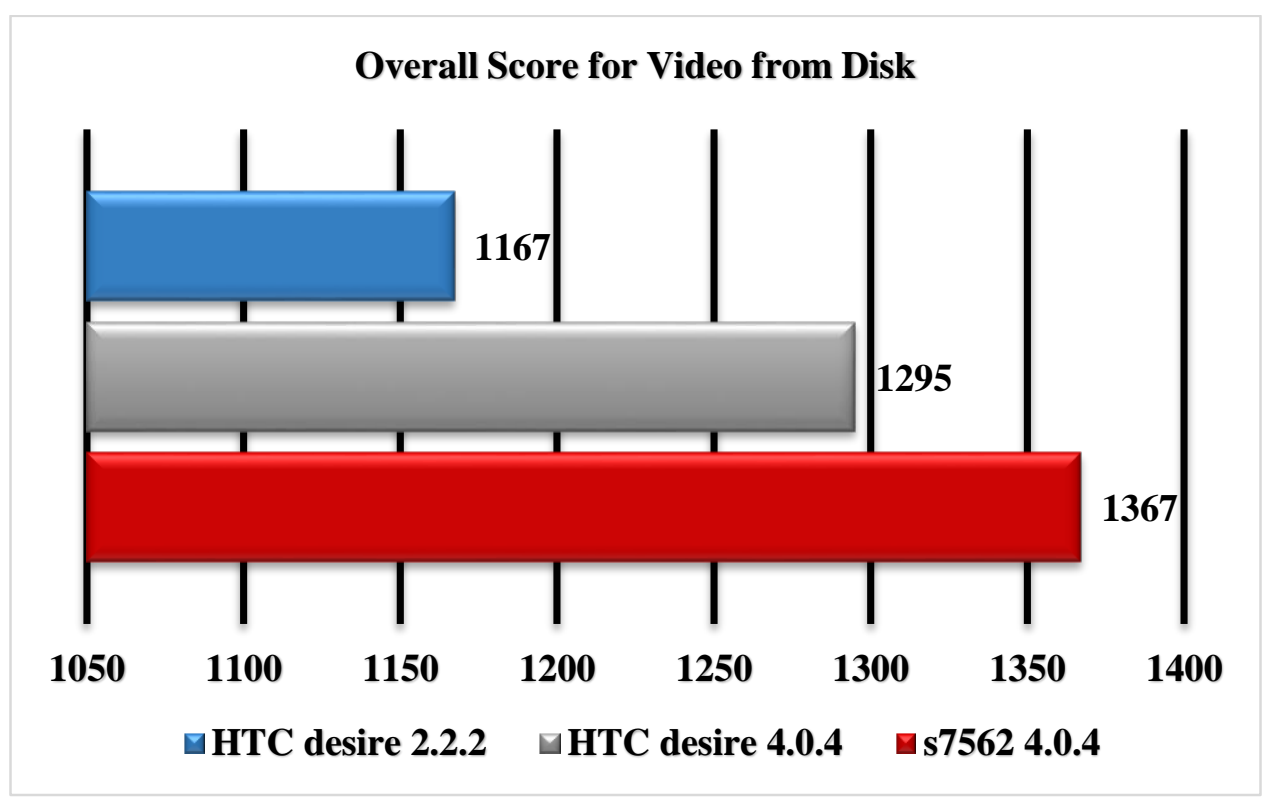

Figure 6c. Playing Video from Disk application. 


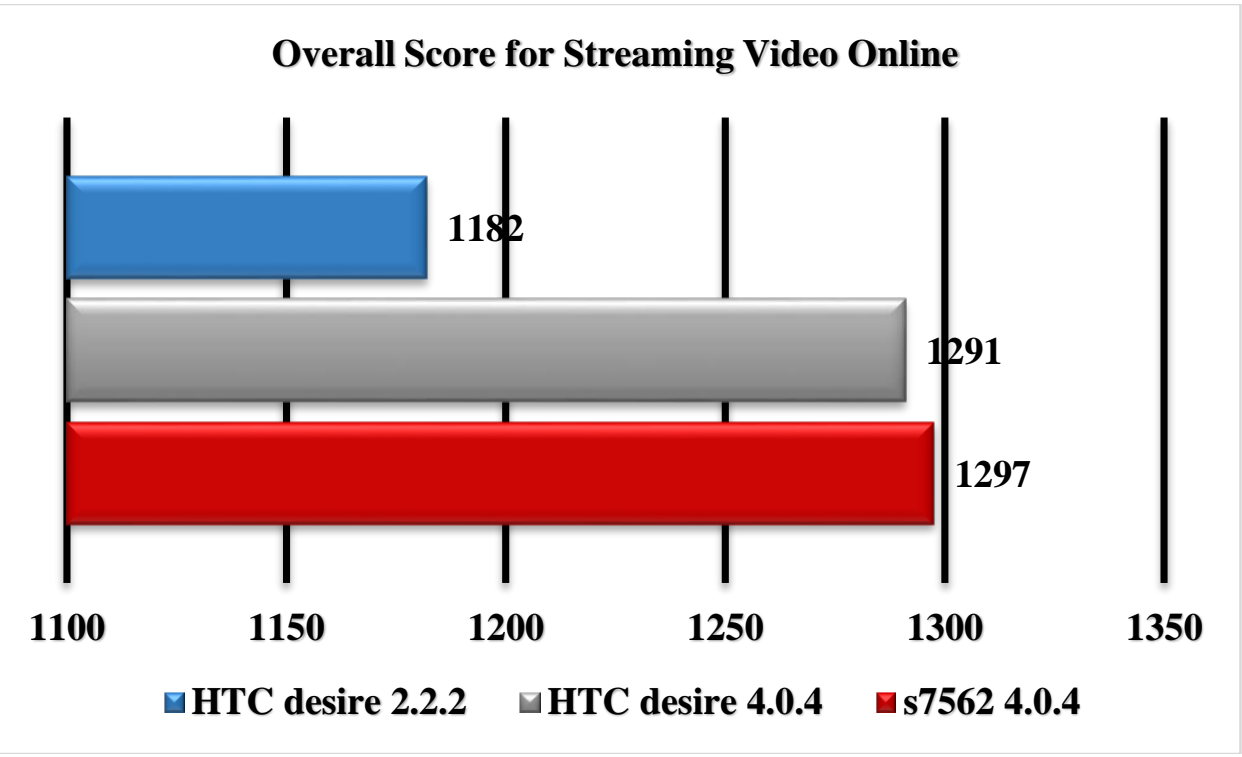

Figure 6d. Streaming Video application.

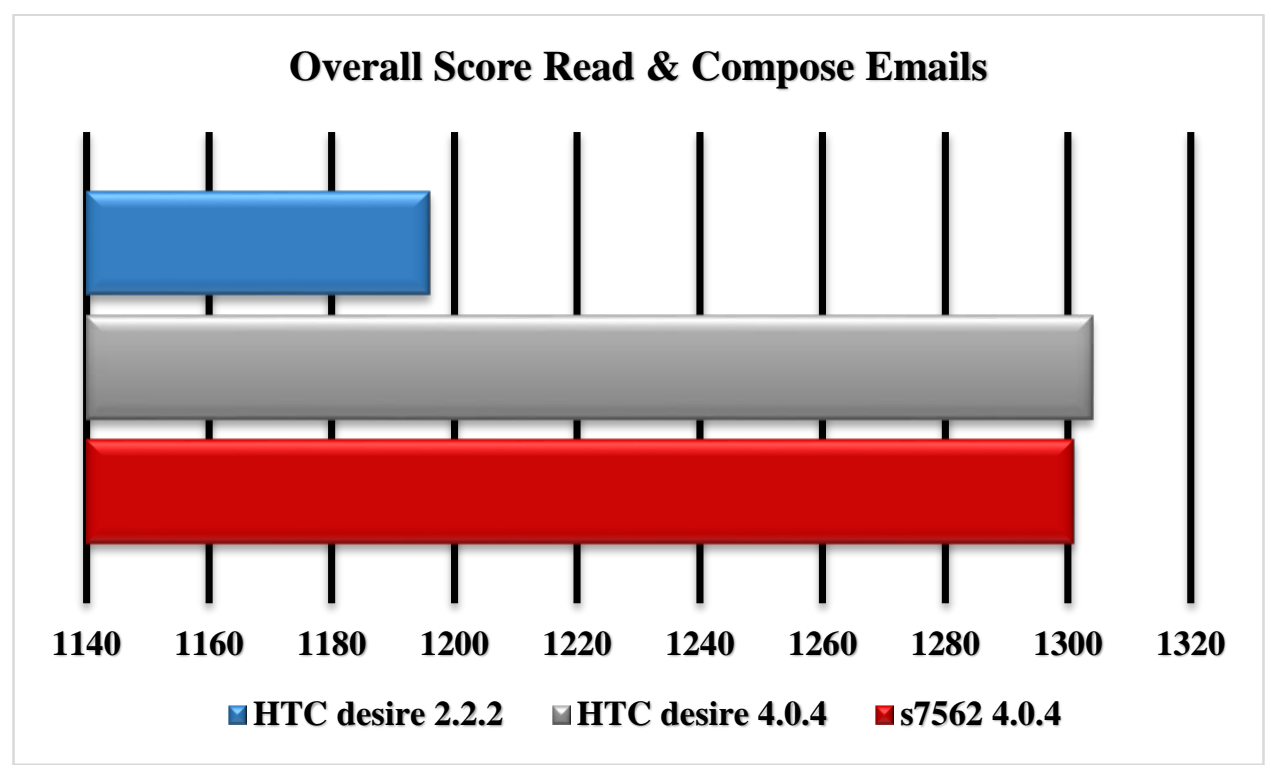

Figure 6e. Email Application. 


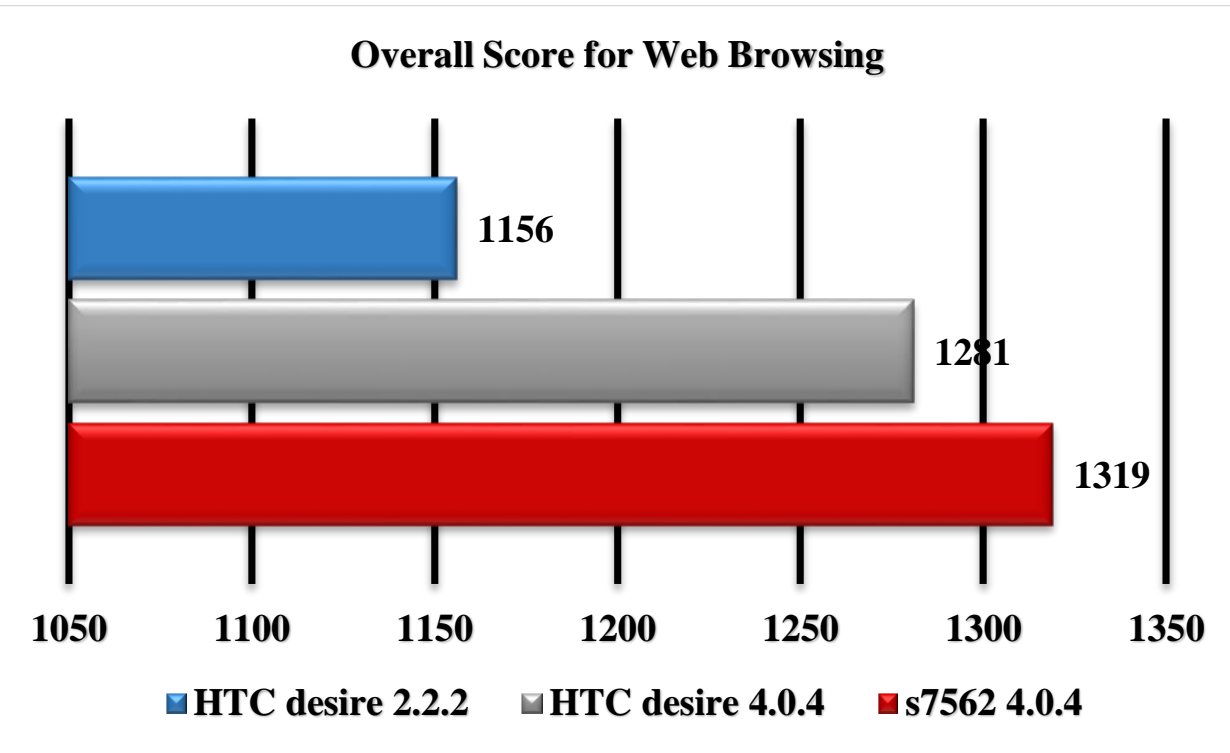

Figure 6f. Web Browsing Application.

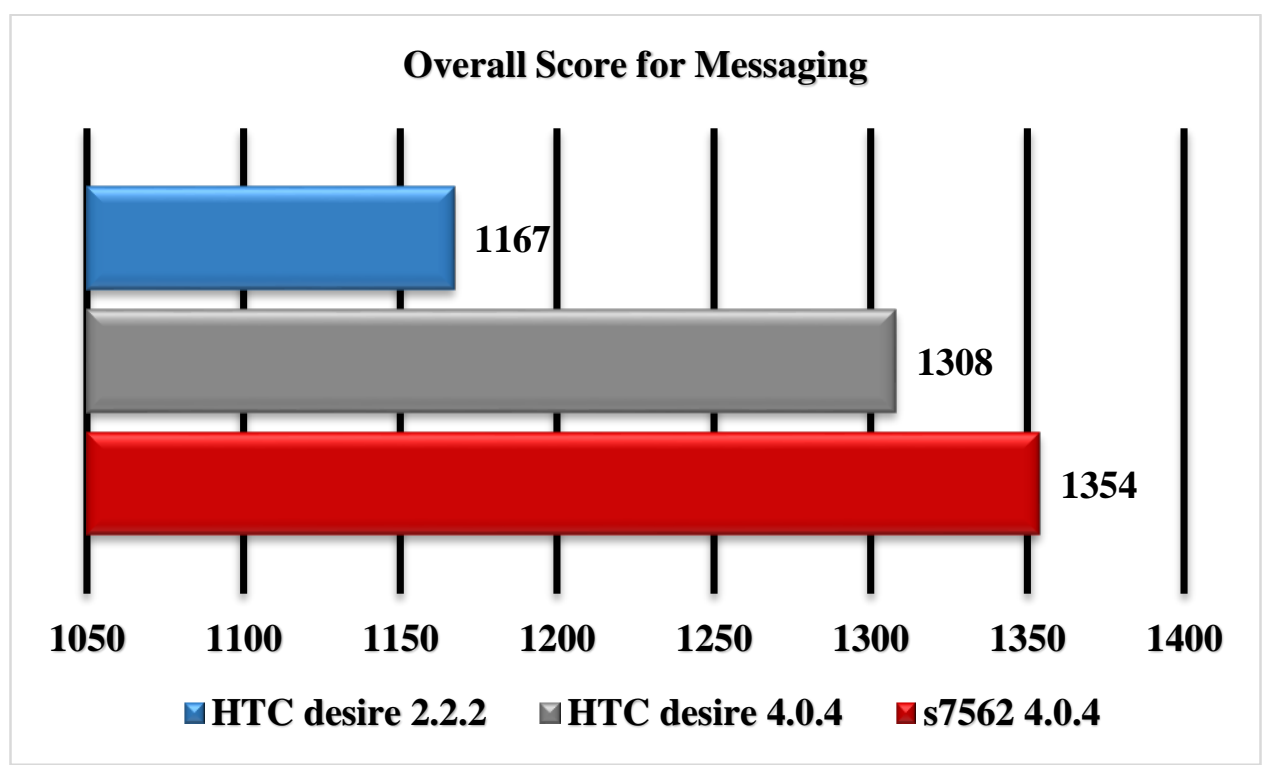

Figure 6g. Messaging Application.

Figure 6. Impact on OS upgrades on various application.

The results shown in Figures 6 indicate that for CPU intensive applications, the relative scores of $\mathrm{OO}, \mathrm{ON}$, and $\mathrm{NN}$ are similar to those seen earlier in Figure 2. However, other applications (email, streaming video, phone calls, messaging) that are less CPU-intensive and more interactive on-screen (requiring graphics usage) seem to benefit the most from OS upgrades 
(with ON scores comparable to NN scores). The graphics capabilities were similar on both devices compared allowing the older device to be better at some applications after the upgrade than the newer device.

\subsection{Discussion}

The main result from the paper is that OS upgrades are beneficial on an older device and provides comparable performance benefits to a newer device if no technological leap separates them. To some extent this is intuitive, but the extensive evaluations in this paper confirm this intuition and provide a quantifiable measure of the benefits of OS upgrades in a way that has never been done before in literature. The intuition that OSs can smooth out the differences in hardware has been expressed in some recent articles such as [12] which talks about the dimishing returns for newer phone models (as seen by the results from this paper) in being enticing to consumers. This trend would help device retention and increase lifespan.

The question of who would perform OS upgrades is an important one. Consumers may lack the technical knowledge to do these upgrades and may have to rely on other entities. There have been recent efforts to refurbish older devices by companies such as Gazelle [14] that buy older devices and resell them. Consumers could elect to pay a service fee to periodically have their devices "refreshed" such as the service offered by iCracked [15]. Modular phones such as those envisioned by Google's Project Ara [6] would further make such efforts easier. Some other articles also note similar trends and embrace the concept of retaining older devices [16].

It is important to keep in mind that there is a limit to using OSs to improve device performance, and older hardware eventually will not work or be supported by newer OS releases. This tends to happen again when technological leaps forces newer OSs to be optimized for those and older hardware components cannot cope up with the demands of these OSs. For example, 
Google's Android OS 4.4.2 recommends at least 1 GB memory while the HTC Desire only has $512 \mathrm{MB}$.

As shown in Figure 7, the limit of benefits from OS upgrades due to hardware limitation was studied.

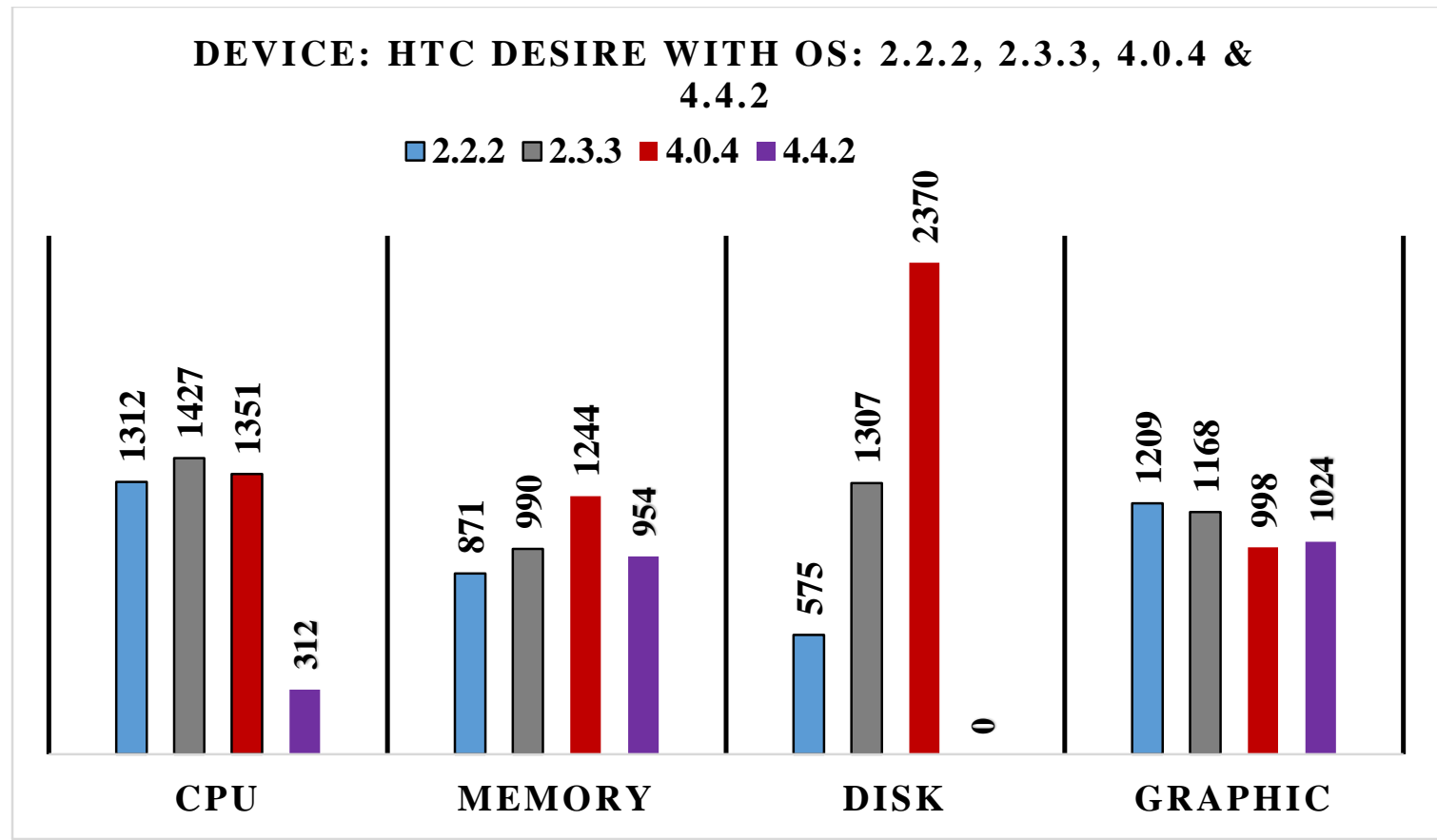

Figure 7. Illustration that OS upgrade benefits are limited to only a few versions before hardware requirements are not met.

The performance of various components improves or remains constant with OS upgrades until the latest release when the trend is towards performance degradation without enough capabilities to meet OS demands. The disk score could not even be computed (hence a score of 0 is assigned) with the Passmark benchmark tool due to lack of resources. The HTC Desire was released in early 2010, while the Android 4.4.2 version was released late 2014. Android 4.3 released in July 2013 had minimum requirements that were met by the HTC Desire device. Thus, one can estimate that OS upgrades cannot provide benefits for devices older than 4 years with current 
trends. With device lifespan averaging less than 2 years currently [8], there is certainly room for improvement by relying increasingly on OS upgrades. 


\section{CHAPTER 4}

\section{CONCLUSIONS \& FUTURE WORK}

\subsection{Conclusions}

The goal of this work was to study the impact of operating system (OS) software upgrades on increase in mobile device lifespan. Towards this broad goal, this work made an attempt to quantify how OS upgrades can bridge the performance gap between older devices and newer devices and motivate users to retain their devices longer and increase device lifespan. The methodology used was to employ a benchmarking tool to compare the performance of an older device to a newer device before and after an OS upgrade. An older device's ability to compete with four other devices with subsequent release dates was studied to better understand the ability of OS upgrades to provide comparable performance against newer releases over time. It was found that an OS upgrade is sufficient to obtain comparable performance to newer devices as long as the newer device does not contain any technology leaps in hardware components. Performance scores for an older device with an OS upgrade were further quantified for seven different applications to better understand how users may perceive the benefits of OS upgrades on older devices compared to a newer device. It was found that OS upgrades were enough to provide comparable performance except for those applications that are $\mathrm{X}$-intensive where $\mathrm{X}$ is a specific hardware component that has improved through a technology leap i.e. the X component is much better in terms of performance on a newer device than the older device. With the time interval between technology leaps typically greater than current device lifespan, it can be concluded that OS upgrades have the potential to increase devices lifespan up to the next technological leap. 


\subsection{Future Works}

Future work in this area would involve conducting usability studies of older devices with an OS upgrade and comparing it to newer devices. This would bring the human element into the picture and will result in incorporating the impact of other components such as display, camera, speakers etc. The benchmarking approach of this paper would still be an useful result to consider even after such a usability study as the latter tends to be highly subjective. 
REFERENCES 


\section{REFERENCES}

[1] R. Puustinen and G. Zadok, "The Green Switch: Designing for Sustain-ability in Mobile Computing," in First USENIX International Workshop on Sustainable Information Technology, February 2010.

[2] P. Somavat, S. Jadhav, and V. Namboodiri, "Accounting for the energy consumption of personal computing including portable devices," in Proceedings of the 1st International Conference on Energy-Efficient Computing and Networking, ser. e-Energy '10, 2010, pp. 141-149.

[3] P. Somavat and V. Namboodiri, "Energy consumption of personal com-puting including portable communication devices," Journal of Green Engineering, vol. 1, no. 4, pp. 447$475,2011$.

[4] M. P. Mills, "The cloud begins with coal. big data, big networks, big infrastructure, and big power. an overview of the electric-ity used by the digital ecosystem," http://www.tech-pundit.com/wp-content/uploads/2013/07/Cloud Begins With Coal.pdf?c761ac.

[5] Nokia, "Sustainability Report," 2011. [Online]. Avail-able: http://www.nokia.com/global/about-nokia/people-and-planet/ strategy/reports/sustainability-reports.

[6] Google, "Project Ara," www.projectara.com/ [cited 15 March 2015].

[7] "Phonebloks - A phone worth keeping," https://phonebloks.com/en/goals [cited 15 March 2015].

[8] V. S. Venkitachalam, V. Namboodiri, S. Joseph, E. Dee, and C. A. B. Jr., "What consumers want in new phones, why, and how often with implications for the environment," IEEE Consumer Electronics Magazine, April, to appear 2015.

[9] S. Joseph, V. Namboodiri, and V. C. Dev, "A market driven framework towards environmentally sustainable mobile computing," SIGMETRICS Perform. Eval. Rev., vol. 42, no. 3, pp. 46-48, 2014.

[10] S. Joseph, V. Namboodiri, and V. Dev, "Towards environmentally sus-tainable mobile computing through an economic framework," Emerging Topics in Computing, IEEE Transactions on, vol. PP, no. 99, pp. 1-1, 2014.

[11] “Passmark Performance Testing," http://www.passmark.com/ [cited 08 September 2014]. 
[12] J. Newman, "Diminishing Returns: The Cold, Hard Truth for CES Smartphones," Time Tech, http://techland.time.com/2013/01/09/diminishing-returns-the-cold-hard-truth-forces-smartphones/.

[13] "Performance Comparison of Android Devices," http://www.androidbenchmark.net/ [cited 05 September 2015].

[14] “Trade-In and Buy Cell Phones,” http://www.gazelle.com/ [cited 25 January 2015].

[15] "Get Back To Life, Fast. Let us come repair or purchase your iPhone, iPad, iPod, or Samsung Device - anytime and anywhere" http://www.iCracked.com/ [cited 21 January 2015].

[16] C. Mims, "In Defense of Good-Enough Gadgets-Why you should re-sist the "cult of the new and shiny' and embrace last years smartphone," The Wall Street Journal. 